OPEN ACCESS

Edited by:

Yolanda López-Vidal, Universidad Nacional Autónoma de México, Mexico

Reviewed by:

Chiyu Zhang,

Fudan University, China

Pragya Dhruv Yadav,

National Institute of Virology (ICMR),

India

*Correspondence:

Zhaoqing Yang

zyang@imbcams.com.cn

Shaohui Ma

shaohuima70@126.com

Specialty section:

This article was submitted to Microbiome in Health and Disease,

a section of the journal

Frontiers in Cellular and

Infection Microbiology

Received: 25 April 2021

Accepted: 26 July 2021

Published: 16 August 2021

Citation:

Liu H, Zhang M, Feng C, Cong S, Xu D, Sun $H$, Yang $Z$ and Ma $S$ (2021)

Characterization of Coxsackievirus A6

Strains Isolated From Children With

Hand, Foot, and Mouth Disease.

Front. Cell. Infect. Microbiol. 11:700191.

doi: 10.3389/fcimb.2021.700191

\section{Characterization of Coxsackievirus A6 Strains Isolated From Children With Hand, Foot, and Mouth Disease}

\author{
Hongbo Liu ${ }^{1,2,3}$, Ming Zhang ${ }^{1,2}$, Changzeng Feng ${ }^{1,2}$, Shanri Cong ${ }^{1,2}$, Danhan $\mathrm{Xu}^{1,2}$, \\ Hao Sun ${ }^{1,2}$, Zhaoqing Yang ${ }^{1,2 *}$ and Shaohui Ma ${ }^{1,2 *}$ \\ 1 Institute of Medical Biology, Chinese Academy of Medical Sciences, and Peking Union Medical College, Kunming, China, \\ 2 Yunnan Key Laboratory of Vaccine Research Development on Severe Infectious Disease, Kunming, China, ${ }^{3}$ Safety \\ Evaluation Center, Sichuan Institute for Drug Control (Sichuan Testing Center of Medical Devices), Chengdu, China
}

Coxsackievirus A6 (CVA6) is a key pathogen causing hand, foot and mouth disease (HFMD). However, there are currently no specific antiviral drugs or vaccines for treating infections caused by CVA6. In this study, human rhabdomyosarcoma (RD), African green monkey kidney (Vero), and human embryonic lung diploid fibroblast (KMB17) cells were used to isolate CVA6 from 327 anal swab and fecal samples obtained during HFMD monitoring between 2009 and 2017. The VP1 genes of the isolates were sequenced and genotyped, and the biological characteristics of the representative CVA6 strains were analyzed. A total of 37 CVA6 strains of the D3 gene subtypes were isolated from RD cells, all of which belonged to the epidemic strains in mainland China. Using the adaptive culture method, 10 KMB17 cell-adapted strains were obtained; however, no Vero cell-adapted strains were acquired. Among the KMB17 cell-adapted strains, only KYN-A1205 caused disease or partial death in suckling mice, and its virulence was stronger than its RD celladapted strain. The pathogenic KYN-A1205 strain caused strong tropism to the muscle tissue and led to pathological changes, including muscle necrosis and nuclear fragmentation in the forelimb and hindlimb. Sequence analysis demonstrated that the KYN-A1205 strain exhibited multiple amino acid mutations after KMB17 cell adaptation. Moreover, it showed strong pathogenicity, good immunogenicity and genetic stability, and could be used as an experimental CVA6 vaccine candidate.

Keywords: Coxsackievirus A6, HFMD, KMB17 cell-adapted strain, biological characteristic, vaccine

\section{INTRODUCTION}

Hand, foot and mouth disease (HFMD) is an infectious childhood illness predominantly caused by enteroviruses (EVs) (Solomon et al., 2010; Weng et al., 2017; Fujimoto, 2018; Zhang H. et al., 2019). EVs have been divided into 15 species, namely EV-A - L and rhinovirus A - C, comprising more than 100 serotypes [http://www.picornaviridae.com]. Notably, EV-A71 and Coxsackievirus A16 (CVA16) are recognized as the key pathogens causing HFMD. The former is almost exclusively associated with severe disease (Xing et al., 2014; Liu et al., 2015). However, since 2008, Coxsackievirus A6 (CVA6), belonging to the EV-A group, has gradually become one of the main 
viruses causing HFMD outbreaks in Europe, the Americas, and Asia (Lott et al., 2013; Montes et al., 2013; Hayman et al., 2014; Ang et al., 2015; Bian et al., 2015; Mirand et al., 2016; Anh et al., 2018). Since 2013, the HFMD etiology monitoring system in many cities in China identified that the CVA6 positivity rate suddenly increased, demonstrating that the virus is one of the key pathogens causing HFMD (Yang et al., 2014; He et al., 2018; Zeng et al., 2018). It has been determined that CVA6 is associated with serious diseases, such as aseptic meningitis, encephalitis and acute flaccid paralysis (Fujimoto, 2018). Li et al. (2018) found that CVA6 accounted for $15.2 \%$ and $16.9 \%$ of severe HFMD cases in China in 2013 and 2015, respectively. Since then, CVA6 has spread throughout mainland China, leading to serious public concern.

CVA6 strains are segregated into 4 genotypes designated as A, $\mathrm{B}, \mathrm{C}$, and D and further subdivided into B1-B2, C1-C2, and D1D3 sub-genotypes. The Gdula prototype strain isolated in the United States in 1949 is classified as genotype A. Genotype B and C strains circulated in mainland China between 1992 and 2007 and in Shandong province of China in 1996 and India in 2008, respectively. Genotype D circulated from 1999 to 2015 in mainland China, Japan, Finland, Spain, and France. B, C, and D Genotypes are further subdivided into B1-2, C1-2, and D1-3 sub-genotypes, respectively. B1 were detected in 1992 in Shandong, China. B2 circulated from 2004 to 2007 in Guangdong, China; D1 and D2 circulated in Japan in 1999 and in France and Spain from 2008 to 2010 and in Japan and mainland of China from 2006 to 2011, respectively. Since 2008, D3 strains gradually become the predominant strains in Finland, Spain, France, Japan, and China (Song et al., 2017; Hoa-Tran et al., 2020).

Intriguingly, it has also been reported that CVA6 is associated with adult HFMD, which has increased in incidence (Broccolo et al., 2019). It has been identified as an important herpes causing pathogen (Shin et al., 2010; Ramirez-Fort et al., 2014); however, the herpes infection is not reported in China's disease monitoring and reporting system. Furthermore, the CVA6 infection differs from typical HFMD (Drago et al., 2017; Balestri et al., 2018). Hence, it has been speculated that the disease burden caused by CVA6 might be significantly underestimated. Unfortunately, at present, no antiviral drugs or vaccines targeting CVA6 are available. Thus, the development of a CVA6 vaccine is essential.

Previous studies demonstrated that CVA6 was difficult to propagate in African green monkey kidney (Vero) and human embryo lung diploid fibroblasts (KMB17, MRC-5) cells (Woods and Young 1988; Prim et al., 2013). The majority of studies utilize human rhabdomyosarcoma (RD) cells to isolate and culture CVA6. Hence, the current research on the CVA6 vaccine primarily focuses on the development of an inactivated vaccine using $\mathrm{RD}$ cells as the cell matrix or a virus-like particle (VLP) vaccine, both of which are retained in mouse model (Yang et al., 2016; Zhou et al., 2016). Unfortunately, the existing formalin-inactivated EV-A71 vaccine shows no crossprotection against CVA6 or other EV infections (Zhu et al., 2014; Yi et al., 2017).
In the present study, the biological characteristics, such as genotype, nucleotide and amino acid differences, one-step growth curve, pathogenicity and immunogenicity of different cell-adapted CVA6 strains were preliminarily investigated to guide future research on the CVA6 vaccine.

\section{MATERIALS AND METHODS}

\section{Virus Isolation and Adaptive Culture}

The stool sample collected from the child was treated using the standard procedure, and adaptively cultured in human rhabdomyosarcoma (RD) cells, African green monkey kidney (Vero) and human embryonic lung diploid fibroblast (KMB17) cells for virus isolation (Prim et al., 2013; Xu and Zhang 2016). When the cells grew into a dense monolayer, the cell monolayer was washed with PBS three times and the filtered and sterilized samples were inoculated on the cell surface. After adsorption for $2 \mathrm{~h}$, an appropriate amount of maintenance solution and mycoplasma removal agent was added, and the cells were moved to $37^{\circ} \mathrm{C}$ incubator containing $5 \% \mathrm{CO}_{2}$ for continuous culture for 7 days. Samples of inducing cytopathic effects (CPE) were considered as positive and were stored at $-20^{\circ} \mathrm{C}$. After three cycles of freeze-thaw, the next round of passage was carried out. This blind passage was performed for three generations. All of the above operations were performed in a BSL-2 biosafety cabinet. The flow chart of the study is provided in Figure 1.

\section{Reverse Transcription Polymerase Chain Reaction (RT-PCR), Sequencing, and Typing}

RT-PCR, sequencing and typing were performed according to a previously described procedure (Liu et al., 2020a). Briefly, the QIAamp Viral RNA Mini Kit (Qiagen, USA) was used to extract the viral RNA from infected cell supernatants. The PrimeScript One Step RT-PCR Kit Ver. 2 (Takara, Dalian, China) was employed to perform RT-PCR. Primers 224 and 222 were used to amplify the partial VP1 sequences. Complete genome fragments were amplified and sequenced using multiple pairs of primers, and the amplification and sequencing primers are summarized in Table S1. The positive amplification products were sequenced by Kunming Qingke Biological Technology Co., Ltd. (Kunming, China). The Enterovirus Genotyping Tool was used for EV classification. The VP1-encoding sequences and complete genomes were compared with sequences available in GenBank using the Basic Local Alignment Search Tool (BLAST) [http://www.ncbi.nlm.nih.gov/BLAST]. The VP1 sequences of 37 CVA6 strains characterized in this study were deposited in the GenBank database (accession numbers: MN514784-MN514822).

\section{Phylogenetic Analysis and Sequence Alignments}

The Molecular Evolutionary Genetic Analysis (MEGA) version 7.0 software with the maximum likelihood (ML) method with 1000 bootstrap replications was used to perform the phylogenetic 


\section{Virus isolation}

human rhabdomyosarcoma (RD) cells , green monkey kidney (Vero) and human embryonic lung diploid fibroblast (KMB17) cells for virus isolation

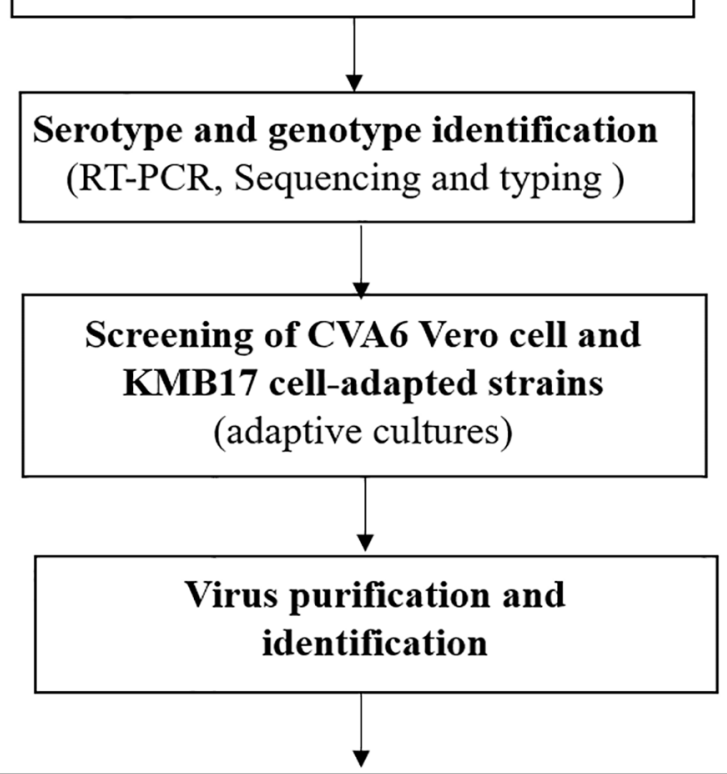

Cell level analysis: one-step growth curve,

Animal level analysis: pathogenicity and immunogenicity,

Molecular level analysis: phylogenetic analysis and sequence alignments.

FIGURE 1 | The flow chart of the study.

analysis of 127 CVA6 strains (i.e., 90 strains from the GenBank database and 37 strains isolated in this work) and CVA10 prototype strain CVA10/Kowali/AF081300 based on the complete VP1 sequence (915 nucleotides) (Fu et al., 2020). CVA6 was genotyped according to previous studies (Song et al., 2017). CVA6 lineage was defined as forming a monophyletic clade and according to a difference of approximately $15 \%$ in the complete VP1 nucleotide sequences was assigned for different genotypes. However, genotypes can be further subdivided into different sub-genotypes, with a difference of over $8 \%$ in VP1 nucleotide sequences (Brown et al., 1999). The Geneious 9.0.2 software was employed for pairwise alignment of these sequences.

\section{Virus Titration}

The virus titres were measured using a microtissue culture technique (MCT) according to a previously described protocol (Zhang et al., 2019). Briefly, serially diluted virus samples were inoculated into RD cells in 96-well plates, which were incubated at $37^{\circ} \mathrm{C}$ in the presence of $5 \% \mathrm{CO}_{2}$ for 7 days. Subsequently, the $50 \%$ cell culture infectious dose $\left(\mathrm{CCID}_{50}\right)$ values were calculated using the Karber method.

\section{Virus Purification}

The CVA6 strains were purified by plaque cloning in RD and KMB17 cells. Briefly, CVA6 strain diluted using a 10-fold gradient $\left(10^{-1}-10^{-5}\right)$ was added to the cell monolayer and incubated at $37^{\circ} \mathrm{C}$ with $5 \% \mathrm{CO}_{2}$ for $2 \mathrm{~h}$. Subsequently, the medium was removed and the cell surface was rinsed three times with phosphate-buffered saline (PBS). The cell surface was then covered with $2 \mathrm{~mL}$ of MEM containing $0.9 \%$ agarose. Following solidification, the inverted culture was incubated at $37^{\circ} \mathrm{C}$ with $5 \% \mathrm{CO}_{2}$. When a single plaque was observed under a microscope, it was absorbed with a pipette and dissolved in $500 \mu \mathrm{L}$ of PBS. The mixture was frozen three times at $-20^{\circ} \mathrm{C}$ and centrifuged at $5,000 \times g$ for $30 \mathrm{~min}$. The supernatant was used to inoculate cells in 24-well plates. When the cells demonstrated $\mathrm{CPE}$, the viral RNA was extracted and identified according to the 
method described above. The virus identified as CVA6 was subjected to next-generation plaque purification. Each strain was purified three times using the same approach.

\section{One-Step Growth Curve of CVA6 Isolates in RD and KMB17 Cells}

Ten CVA6 RD cell-adapted strains and KMB17 cell-adapted strains were inoculated into RD and KMB17 cells with $\mathrm{MOI}=1$. Three replicates were performed at each time point. After inoculation, the virus solution was harvested every $24 \mathrm{~h}$ for 8 days. The virus titres were determined according to the method described above. The one-step growth curve of the virus was plotted as the sample collection time (abscissa) vs. virus infection titres (ordinate).

\section{Experimental Animal Infections}

Seven CVA6 RD cell-adapted strains with highly infectious titres and their KMB17 cell-adapted strains were selected and inoculated into the cranial cavity of one-day-old BALB/c suckling mice at a dose of $6.5 \operatorname{lgCCID}{ }_{50}$, and the mice were monitored for 15 days. Specific pathogen-free BALB/c suckling mice were purchased from Hunan Slack Jingda Experimental Animal Co., Ltd., Hunan, China. The experimental mice were infected with the viral stock supernatant by intracranial injection, while the negative control mice were mock infected with uninfected cell supernatant via the same route $(30 \mu \mathrm{L} /$ per mouse, 6-10 mice in each group). The pathogenicity of the strain was evaluated based on the clinical characteristics of neonatal mice. The pathogenic strain was selected and inoculated into the cranial cavity of the neonatal mice at $6.5,5.5$, and $4.5 \operatorname{lgCCID}_{50}$. The pathogenicity of the strain before and after KMB17 cell adaptation was compared based on the average body weight, clinical score, survival rate, hematoxylin and eosin (HE) staining, immunohistochemistry results and viral load in each tissue.

\section{Histopathological and Immunohistochemistry (IHC) Analyses}

The histopathological and immunohistochemistry (IHC) analyses were performed according to previously reported methods. Briefly, the tissues of the neonatal mice were dissected and placed in $4 \%$ formalin for a week. Following fixation, the tissue sections were embedded in paraffin and stained with HE. After dehydration, the tissue samples were mounted with neutral gum. For IHC analysis, mouse anti-CVA6 antibody (1:1,000 dilution) was incubated at $4^{\circ} \mathrm{C}$ overnight. Peroxidase-conjugated secondary antibody was added for $50 \mathrm{~min}$ at room temperature and then developed using the diaminobenzidine tetrahydrochloride developer solution. All sections were examined under a microscope slide scanner (3D HISTECH Pannoramic 250, Hungary). The acquired images were collected and analyzed.

\section{Tissue Sampling and RT-qPCR}

The heart, liver, spleen, lung, kidney, small intestine and brain tissues of the suckling mice were weighed and ground with a high speed grinder (KZ-II, Servicebio, Wuhan, China). The total RNA was extracted using the TRIzol reagent (Invitrogen, USA). Subsequently, real-time PCR was performed utilizing the One
Step PrimeScript ${ }^{\text {TM }}$ RT-PCR Kit (Takara) according to the manufacturer's protocol. Primers CVA6-qP-F (5' TACCACCGGGARAAACGTCCACG-3') and CVA6-qP-R (5'CGGTCAGYTGCAGTGTTAGT-3') as well as CVA6-probe (FAM-ACGTGAGAGCTTGGGTACMTAGACCCCTTCBHQ) were used. A sequence of 1468 bp including VP1 fragments amplified by RT-PCR with the primers CVA6VP1-F (5'-TAATACGACTCACTATAGGGGGTCAGAT CTGCAGGTATTAC-3') and CVA6-VP1-R (5'-GAGGACACC AGAAGATCTCG-3') was inserted into the pMD18-T plasmid. The recombinant plasmid was further linearized. VP1 RNA obtained from the in vitro transcription was then used to evaluate the copies of CVA6.

\section{Neutralization Assays}

The neutralization assays were conducted according to previously described methods. The RD cells $\left(1 \times 10^{5} / \mathrm{mL}\right)$ were seeded in 96-well microplates. The serum was serially diluted two-fold by mixing with equal volumes of the CVA6 strains (100 $\left.\mathrm{CCID}_{50}\right)$ at $37^{\circ} \mathrm{C}$ for $1 \mathrm{~h}$. The $\mathrm{RD}$ cells were inoculated with the virus-serum mixtures and incubated at $37^{\circ} \mathrm{C}$ for 7 days.

\section{RESULTS}

\section{Primary Characterisation of the Virus Isolates}

The RD, Vero and KMB17 cells were used to isolate viruses from clinical samples. The virus serotype identification was conducted according to a previously reported approach (Du et al., 2019). A total of 37 CVA6 strains were only isolated from RD cells between 2009 and 2017 during HFMD monitoring in the Yunnan and Hubei provinces, China, but failed to from KMB17 and Vero cells. And by ten adaptive cultures, no CVA6 strain was recovered from Vero and KMB17 cell culture, respectively. 37 CVA6 strains isolated from clinical samples were provided in Table S2. The complete VP1 sequences of the 37 strains described in this study had be deposited in the GenBank database under the accession numbers MN514784 to 514822 . All isolates belonged to the D3 gene subtype (Figure 2). The nucleotide and amino acid homologies of the VP1 sequences were $95.8-100 \%$ and $97.0-100 \%$, respectively. After the Vero and KMB17 cells were independently adapted, 10 CVA6 KMB17 celladapted strains were obtained and denoted as KYN-A5, KYNA110, KYN-A1205, KYN-N15, KXY4051, KYN-A13, KYN-A100, KYN-A129, KYN-A68, and KYN-A2. However, no CVA6 Vero cell-adapted strains were acquired. The polyclonal anti-KYNA1205 antiserum completely neutralized nine CVA6 KMB17 celladapted strains (the highest neutralization titre reached 1:1024), indicating that the strains exhibited good immunogenicity.

\section{One-Step Growth Curve of Different Cell-Adapted Strains}

The one-step growth curve of these high titre $\mathrm{RD}$ cell-adapted strains and their KMB17 cell-adapted strains, were plotted (Figure 3). It is noteworthy that at each time point, the virus titres of the YN-A129, YN-A68 and YN-A2 strains in the two studied cell lines were lower than the titres of the other seven 


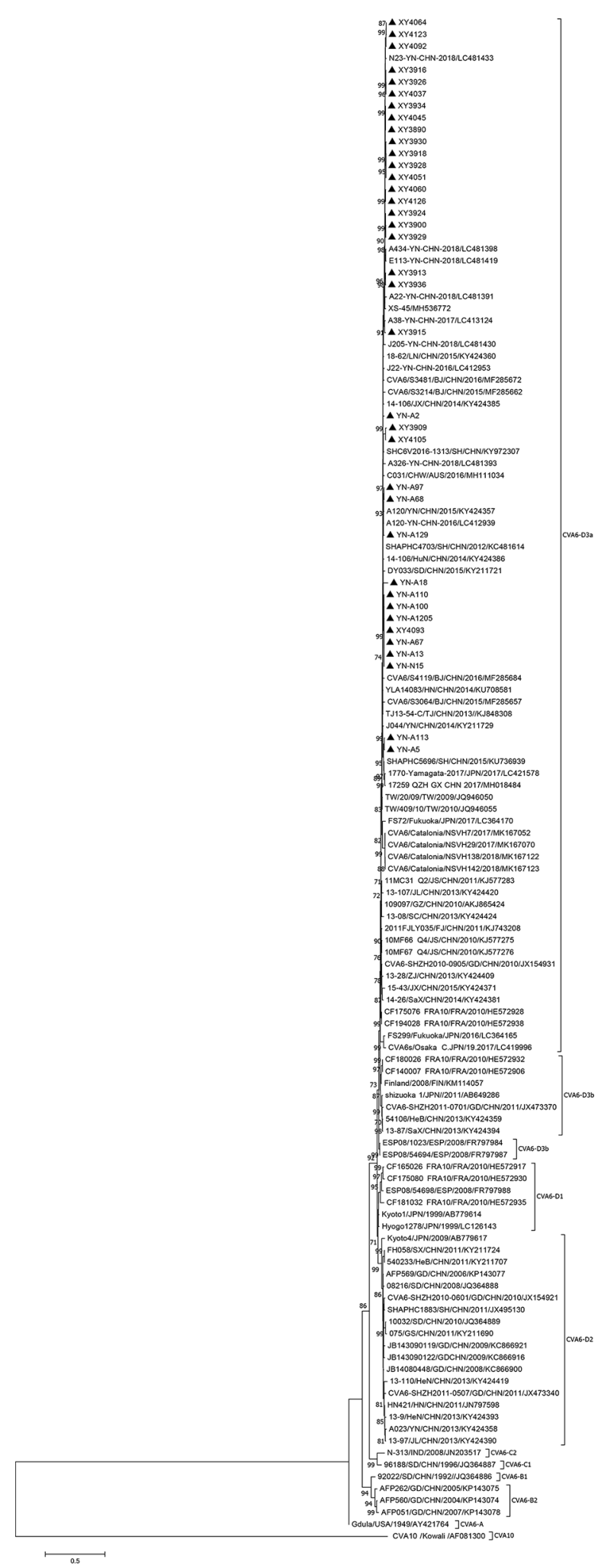

FIGURE 2 | Phylogenetic analysis based on full-length VP1 of CVA6. The 37 CVA6 isolates from this study and 90 representative strains of CVA6 from GenBank and CVA10 prototype strain CVA10/Kowali/AF081300 were used to construct phylogenetic trees based on the full-length VP1 sequence (915 nucleotides) (Test of Phylogeny: Bootstrap method, number of Bootstrap Replications: 1,000 Mode: Kimura 2-parameter model), showing a bootstrap value of $>70 \%$. $\mathbf{\Delta}$ indicates the CVA6 strain isolated in this study. 
strains. By pairwise comparisons of the nucleotide and amino acid sequences of the three whole genomes, no specific mutations were found. Thus, the seven strains with higher titres $(>6.0$ $\log C \mathrm{CID}_{50}$ ) were used in the subsequent investigations.

\section{Pathogenicity Analysis}

Among the seven selected CVA6 RD cell-adapted strains, the RYN-A1205, RYN-N15, RYN-A13 and RXY4051 strains were pathogenic to suckling mice, with the RXY4051 strain exhibiting the strongest virulence (Figure S1). The course of morbidity was comparable. Decreased vitality, limb weakness and paralysis of the forelimb or hindlimb muscles were observed. Moreover, among the CVA6 KMB17 cell-adapted strains, only KYNA1205 caused clinical symptoms in suckling mice (Figure S2). Hence, the RYN-A1205 strain and its KMB17 cell-adapted strain were inoculated into the cranial cavities of 1-day-old BALB/c suckling mice. The results demonstrated that the RYN-A1205 strain had an increase in virulence after the KMB17adaption (Figure 4).

\section{Immunohistochemical and Histopathological Analyses}

When the clinical score reached grades $4-5$, the newborn mice were sacrificed and their heart, liver, spleen, lung, kidney, intestine, brain, forelimb muscle and hindlimb muscle tissues were taken for IHC and HE staining. The results revealed that after infection with the RYN-A1205 and KYN-A1205 strains, the number of leukocytes in the liver sinus increased and minor infiltration of neutrophils was noted (Figure 5). Moreover, large extramedullary hematopoietic foci in the red pulp and multinucleated giant cells were detected. The alveolar wall was considerably thicker due to infiltration of numerous lymphocytes and neutrophils. The number of large areas of muscle fibres in the forelimb muscle and hindlimb tissues decreased. The muscle fibres appeared necrotic, mitotic or dissolved, which was accompanied by increased lymphocyte and neutrophil infiltration. Additionally, a large number of fibroblasts and some hyperplastic connective tissues were observed. Other tissues showed no significant pathological changes. The kidney, lung, small intestine, forelimb muscle and hindlimb muscle tissues displayed distinct CVA6 antigen distribution, which was evidenced by dark brown staining color. In contrast, the remaining tissues exhibited no antigen distribution.

\section{Tissue Viral Loads in CVA6-Infected Mice}

Both RYN-A1205 and KYN-A1205 strains were inoculated into the brains of suckling mice at a dose of $5.5 \mathrm{lg} \mathrm{CCID}_{50}$. The viral loads of the heart, liver, spleen, lung, kidney, intestine, brain, forelimb muscles, hindlimb muscles of the suckling mice were measured on the 4 th, 8 th and 12th days after infection. The
A
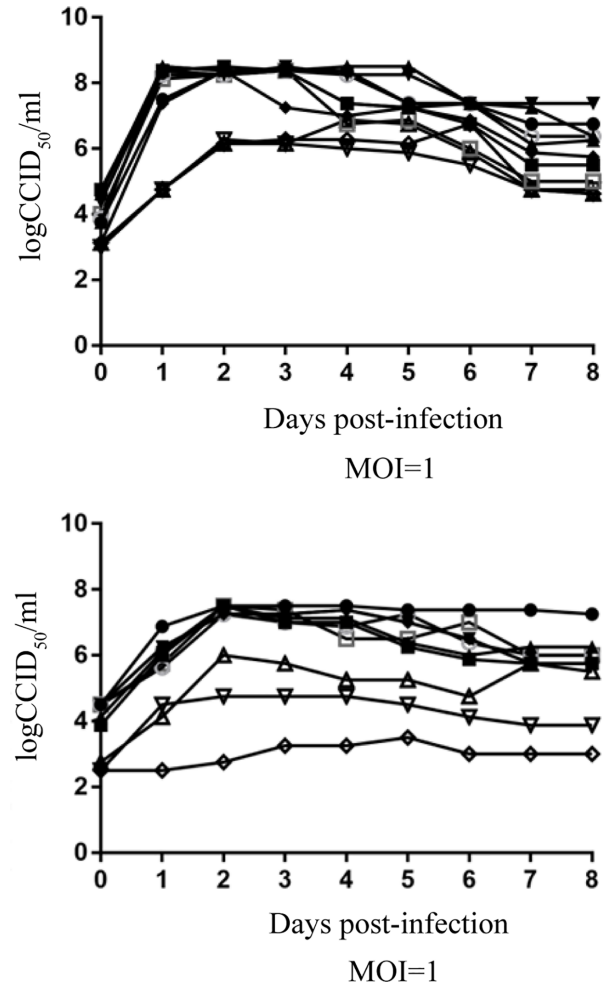

$\rightarrow$ RYN-A5

$\rightarrow$ RYN-A110

$\star$ RYN-A1205

$\rightarrow$ RYN-N15

$\rightarrow$ RXY4051

-๑ RYN-A13

$\because$ - RYN-A100

$\triangle$ RYN-A129

$\rightarrow$ RYN-A68

$\rightarrow$ RYN-A2

FIGURE 3 | The one-step growth curve of these high-titre RD cell-adapted strains and their KMB17 cell-adapted strains. Panel (A) (RD cell lines) and Panel (B) (KMB17 cell lines). 


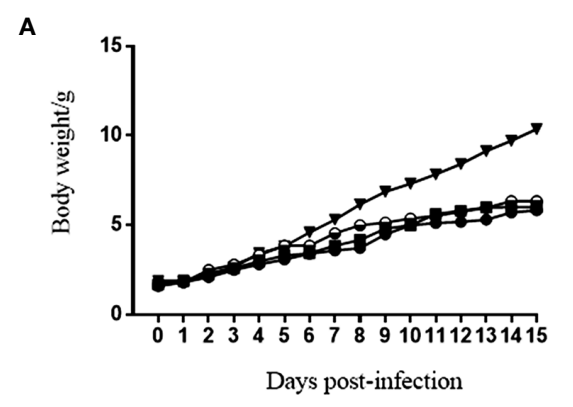

C

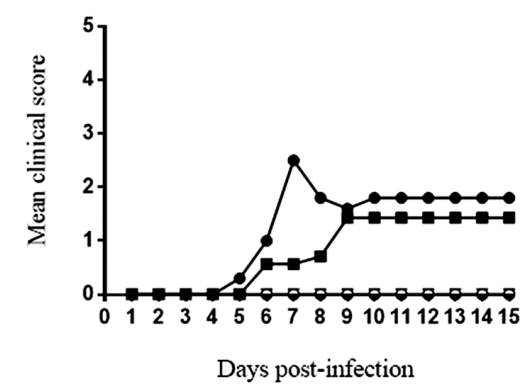

E

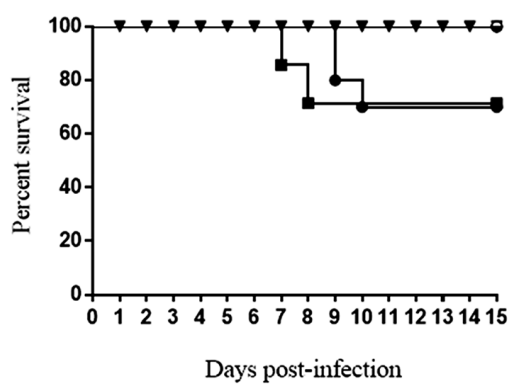

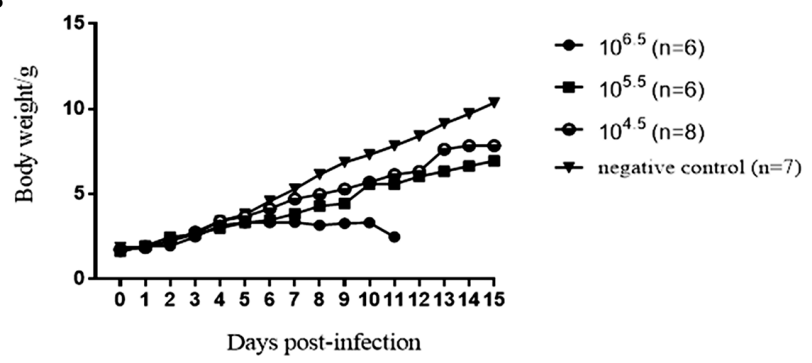

D

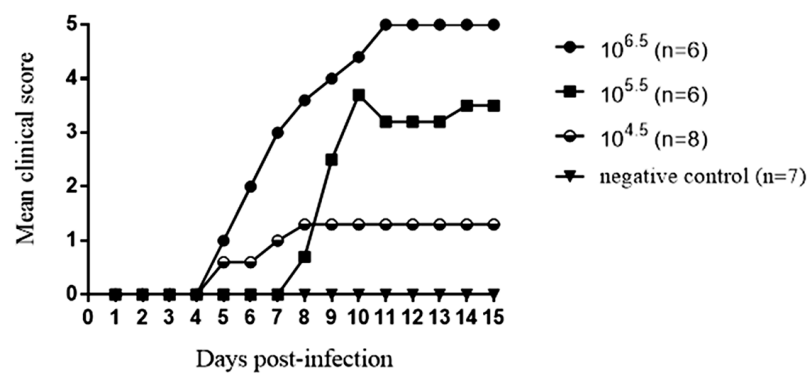

$\mathbf{F}$

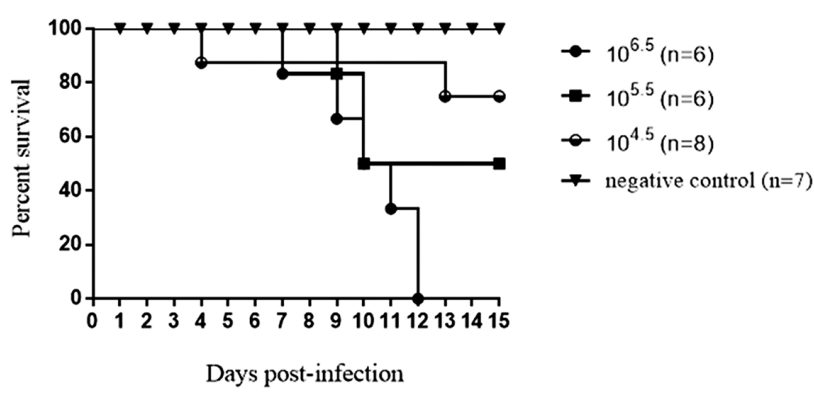

FIGURE 4 | Comparison of virulence of the RYN-A1205 and KYN-A1205 strains. The body weight, mean clinical score and percent survival of neonatal mice injected with the RYN-A1205 strain (A, C, E). The mean clinical score and percent survival of neonatal mice injected with the KYN-A1205 strain (B, D, F). Negative control mice were administered an equal volume of an uninfected cell supernatant instead of the virus.

results showed that there was no significant difference in the viral load of this strain before and after the KMB17 cell adaptation, and all of the suckling mouse tissues showed strong muscle phagocytosis (Figure 6). The virus load of the front and hind limbs of the suckling mouse was the highest on the 8th day post infection (dpi) among all tissues.

\section{Sequence Analysis of Different Cell-Adapted Strains}

The complete genome of the RXY4051, KXY4051, RYN-A1205, and KYN-A1205 strains were obtained by Sanger sequencing (accession numbers: MT364500, MT364501, MN184852, MN184852, respectively). The full-length genomes of these four strains are $7351 \mathrm{nt}, 7352 \mathrm{nt}, 7442 \mathrm{nt}$, and $7397 \mathrm{nt}$, the 5'UTR is 696 nt, $697 \mathrm{nt}, 748 \mathrm{nt}$, and $748 \mathrm{nt}$, and the 3 'UTR is $49 \mathrm{nt}, 50 \mathrm{nt}, 91 \mathrm{nt}$, and $46 \mathrm{nt}$, respectively. All contain an open reading frame of 6606 nucleotides, encoding a polyprotein containing 2201 amino acids. P1 structural protein has 870 amino acids, and P2 and P3 non- structural proteins encode 578 and 753 amino acids, respectively. Among them, the complete genome nucleotide and amino acid homology of the RXY4051 and KXY051 strains were $94.2 \%$ and 97.9\%, respectively (Table S3). There are 48 amino acid differences between these two strains (Table S4), five amino acid differences in the VP2 region; four amino acid mutations in the VP3 region; and ten amino acid differences in the VP1 region. There are three, three, two, two, eighteen amino acid differences in $2 \mathrm{~A}, 2 \mathrm{~B}, 3 \mathrm{~A}, 3 \mathrm{C}$, and $3 \mathrm{D}$, respectively. In addition, the NCBI BLAST tool was used to compare the strains with the most homologous nucleotide sequences to the respective gene regions of the RXY4051 and KXY4051 strains (Table S5). The results showed that the complete genomes of RXY4051 and KXY4051 strains were the most homologous to CVA6/XS45/MH536772 (98.30\%) and CVA6/S2792/BJ/CHN/2014/MF285648 (98.35\%), respectively, and other gene regions were most homologous to the CVA6 strains, suggesting that the two strains have not recombined with other serotype viruses. In addition, the complete genome nucleotide 


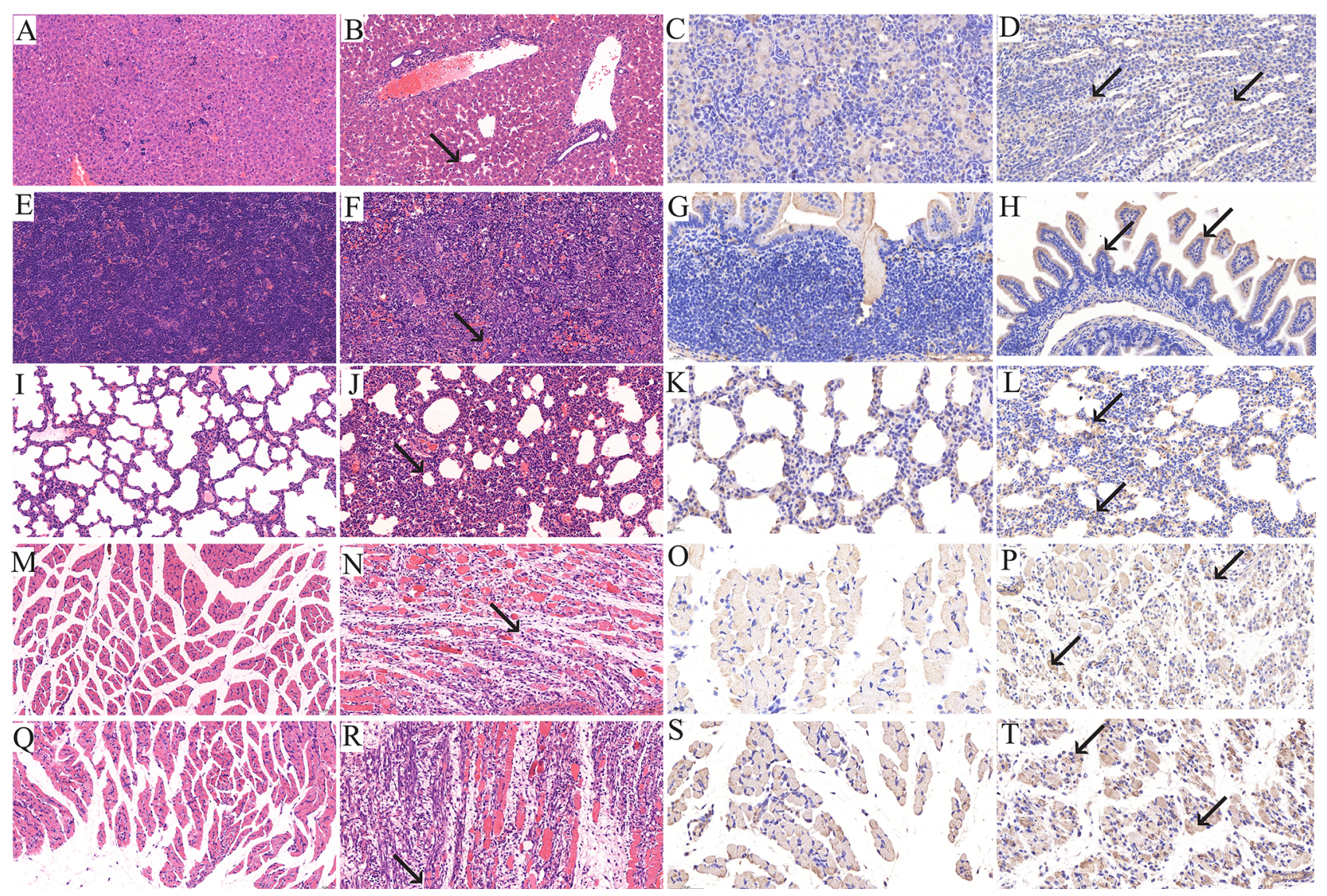

FIGURE 5 | Histopathological (×200) and immunohistopathological analyses of tissues isolated from CVA6-infected neonatal mice (×400). One-day-old BALB/c mice were intracranially injected with $5.5 \mathrm{lgCCID}_{50}$ of the KYN-A1205 strain or an equal volume of uninfected cell supernatant. Obvious histological changes in the liver, spleen, lung, forelimb muscle and hindlimb muscle tissues of the experimental group (B, F, J, N, R, black arrow) were noted. No histological changes were observed in the liver, spleen, lung, forelimb muscle and hindlimb muscle tissues of the negative control group (A, E, I, M, Q). The CVA6 antigen was detected in the liver, lung, intestine, forelimb muscle and hindlimb muscle tissues of the experimental group (D, H, L, $\mathbf{P}, \mathbf{T}$, black arrow). No antigen was detected in the liver, lung, intestine, forelimb muscle and hindlimb muscle tissues of the negative control group $(\mathbf{C}, \mathbf{G}, \mathbf{K}, \mathbf{O}, \mathbf{S})$.

and amino acid sequence homology of the RYN-A1205 and KYNA1205 strains were $99.80 \%$ and $99.40 \%$, respectively. Compared with the RYN-A1205 strain, a total of 15 nucleotide mutations occurred in the strain KYN-A1205, resulting in 12 amino acid substitutions (Table S6).

\section{DISCUSSION}

Following the adoption of the EV-A71 vaccine, the virus spectrum of HFMD has gradually changed. Recently, CVA6 has become the main pathogenic serotype in several regions. CVA6 strains have been segregated into A, B, C, and D, and B1B2, C1-C2, and D1-D3 subgenotypes. Since 2008, genotype D3 strain gradually becomes the main epidemic strain worldwide. In addition, although sporadic substitutions were found among a few CVA6 epidemic strains, the amino acid sequences were highly conservative by predicted putative functional loops located in the VP4, VP2, VP3, and VP1 of CVA6 (Hoa-Tran et al., 2020).
And Loop prediction is consistent with neutralizing linear epitopes from previous report (Xu et al., 2017). Thus, D3 strain is a candidate for developing a CVA6 vaccine (Hoa-Tran et al., 2020).

The CVA6 D3 subtype epidemic strains exhibit stronger transmission ability, infectivity and virulence, which might be associated with a CVA6 epidemic (Song et al., 2017). Based on the nucleotide sequence alignment and phylogenetic analysis of the VP1 sequences, all CVA6 isolates evaluated in the present study were of the D3 subtype, which is consistent with the epidemic strains from mainland China and most international isolates. Hence, it was hypothesized that in-depth research into the known CVA6 isolates would provide crucial guidance for the rational design of effective vaccines against CVA6 infection.

The use of cell lines is necessary for the isolation, proliferation and detection of viruses as well as for the production of viral vaccines. Previous studies demonstrated that proliferation of CVA6 on cell matrices produced by Vero, MRC-5 and other cell lines commonly used during vaccine development was challenging (Woods and Young 1988; Prim et al., 2013). At 


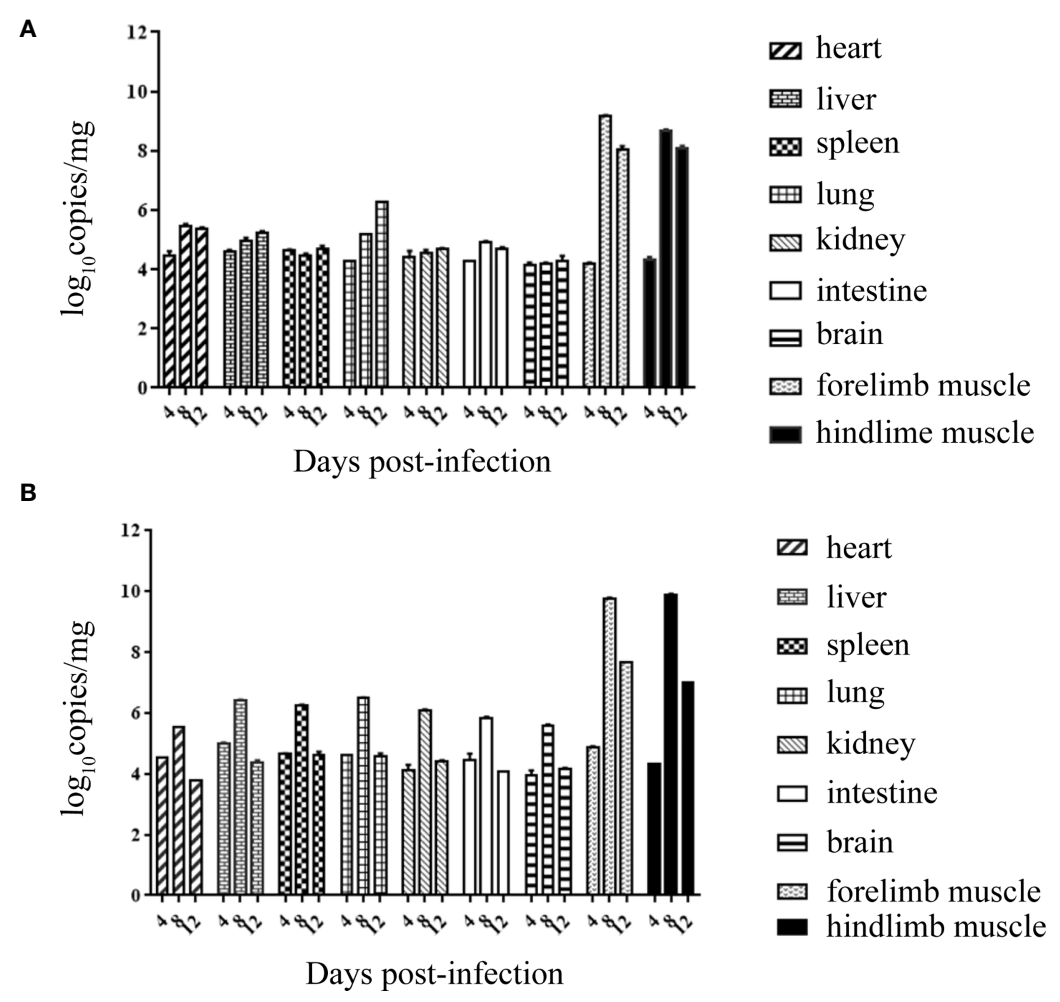

FIGURE 6 | Viral loads of all tissues of suckling mice on the 4th, 8th and 12th dpi with the RYN-A1205 (A) and KYN-A1205 (B) strains.

present, the majority of studies use RD cells to isolate and culture CVA6, which has hindered the development of effective CVA6 inactivated vaccines. Continuous passage is a traditional adaptive screening method, which has been widely employed to screen various viral adaptive strains (e.g. EV-A71, rabies virus, rotavirus, poliovirus and hepatitis A virus). In this work, 37 CVA6 strains were isolated from $\mathrm{RD}$ cells, while, isolation from Vero and KMB17 cells was unsuccessful. Following adaptive culture, only 10 CVA6 KMB17 cell-adapted strains were acquired. This was attributed to the changes in the living environment of the virus after infecting different hosts. Moreover, it is known that CVA6, CVA10, EV-A71 and CVA16 are characterized by high amino acid homology ( 67\%) (Xu et al., 2017). As for CVA6, it is problematic to adapt to Vero and KMB17 cells, which may be related to the inefficient internal ribosome entry site (IRES) in directing translation, its inability to induce the cellular protein synthesis shutoff, and its deviated codon usage with respect to the cell codon usage. Altogether, this may result in competition for the translational machinery and tRNAs, which additionally may not be well adapted to the virus requirements, contributing to the modest productive growth of CVA6 in cell culture (ChavarriaMiró et al., 2021). In addition, host cell receptors are critical for virus invasion and adherence. Previous studies revealed that scavenger receptor class B, member 2 (SCARB2) and P-selectin glycoprotein ligand-1 (PSGL-1) are the specific receptors for EVA71 and CVA16, whereas KREMEN1 is a host cell receptor for
CVA6 and CVA10 (Nishimura et al., 2009; Yamayoshi et al., 2009; Zhu et al., 2018). The surface loops of VP1 (i.e., BC, DE, EF and $\mathrm{HI}$ loops) are the preferred binding sites for numerous small RNA virus receptors. The significant differences in the capsid protein structures in these four loops have been previously reported for CVA6, EV-A71 and CVA16. Hence, the varying arrangement of these four loops could result in many unique capsid surfaces, which might explain why CVA6, EV-A71 and CVA16 do not share the same receptors.

It is recognized that animal models are crucial for vaccine research. Yang et al. found that the RD cell-adapted strain CA6/ 141 showed a strong tendency towards skeletal muscle and skin (Yang et al., 2016). Large antigen distribution was detected in the skeletal muscles of the hindlimbs and spine. In addition, Zhang et al. reported that the CVA6 RD cell-adapted strain WF057R caused significant pathological changes in the brain, hind skeletal muscles and lungs of infected neonates (Zhang et al., 2017). In the current study, three representative strains exhibiting high pathogenicity caused obvious damage to the forelimb and hindlimb muscles. Furthermore, RYN-A1205 and KYN-A1205 caused damage to the liver, spleen and lungs of the infected mice. These outcomes demonstrated that the CVA6 strain showed strong tropism to the muscles of suckling mice, which might lead to paralysis of the newborn animals and an inability to obtain breast milk, thereby accelerating their death. Other pathological changes might be related to the age of the experimental mice, virus strain, inoculation route and dose. Compared with EV-A71 and 
CVA16, CVA6 showed weaker tropism to the nervous system and myocardium of suckling mice, which was consistent with the previously reported clinical findings. EV-A71 and CVA16 are typically associated with severe HFMD and nervous system complications; however, severe HFMD caused by CVA6 is rare.

VP1 plays an important role in the virulence and immunogenicity of EVs. The change of the VP1 protein may be related to the symptoms seen in the nervous system caused by EV-A71 infection (Zhang et al., 2014). Similarly, through KMB17 cell adaptation, the 90th, 140th, 240th and 305th amino acids of the VP1 coding region of the RYN-A1205 strain were mutated, and the 305th amino acid substitution was located in the epitope of the CVA6 antigen. The effect of the change at this amino acid position on the immunogenicity of the RYN-A1205 strain requires further examination.

Evaluation of virus strain immunogenicity is critical to vaccine research. The results of the present study revealed that the maximum neutralizing titre of a polyclonal antiserum of the KYN-A1205 strain reached 1:1024, which completely neutralized nine other KMB17 cell-adapted strains. This indicated that KYN-A1205 displayed good and stable immunogenicity. Moreover, in our previous research, KYN-A1205 was passed to the 15th generation in KMB17 cells. The nucleotide and amino acid sequence homology of the P1, P5, P10 and P15 generation subviruses was established at $99.97 \%-100 \%$ and $99.90-100 \%$, respectively, indicating good genetic stability (Liu et al., 2020b). In summary, we identified a CVA6 KMB17 cell-adapted strain KYN-A1205 with good pathogenicity and immunogenicity. KYN-A1205 could be a promising candidate strain for the development of a CVA6 vaccine.

\section{DATA AVAILABILITY STATEMENT}

The datasets presented in this study can be found in online repositories. The names of the repository/repositories and accession number(s) can be found in the article/Supplementary Material.

\section{REFERENCES}

Ang, L. W., Tay, J., Phoon, M. C., Hsu, J. P., Cutter, J., James, L., et al. (2015). Seroepidemiology of Coxsackievirus A6, Coxsackievirus A16, and Enterovirus 71 Infections Among Children and Adolescents in Singapore 2008-2010. PloS One 10, e0127999. doi: 10.1371/journal.pone.0127999

Anh, N. T., Nhu, L. N. T., Van, H. M. T., Hong, N. T. T., Thanh, T. T., Hang, V. T. T., et al. (2018). Emerging Coxsackievirus A6 Causing Hand, Foot and Mouth Disease, Vietnam. Emerg. Infect. Dis. 24, 654-662. doi: 10.3201/eid2404.171298

Balestri, R., Bellino, M., Landini, L., Tasin, L., Rizzoli, L., Speziali, L., et al. (2018). Atypical Presentation of Enterovirus Infection in Adults: Outbreak of 'Hand, Foot, Mouth and Scalp Disease' in Northern Italy. J. Eur. Acad. Dermatol. Venereol 32, e60-e61. doi: 10.1111/jdv.14507

Bian, L., Wang, Y., Yao, X., Mao, Q., Xu, M., and Liang, Z. (2015). Coxsackievirus A6: A New Emerging Pathogen Causing Hand, Foot and Mouth Disease Outbreaks Worldwide. Expert Rev. Anti Infect. Ther. 13, 1061-1071. doi: 10.1586/14787210.2015.1058156

Broccolo, F., Drago, F., Ciccarese, G., Genoni, A., Puggioni, A., Rosa, G. M., et al. (2019). Severe Atypical Hand-Foot-and-Mouth Disease in Adults Due to Coxsackievirus A6: Clinical Presentation and Phylogenesis of CV-A6 Strains. J. Clin. Virol. 110, 1-6. doi: 10.1016/j.jcv.2018.11.003

\section{ETHICS STATEMENT}

The studies involving human participants were reviewed and approved by the Human Ethics Commission at the Institute of Medical Biology, Chinese Academy of Medical Sciences. Written informed consent to participate in this study was provided by the participants' legal guardian/next of kin. The animal study was reviewed and approved by the animal ethics committee, Institute of Medical Biology, Chinese Academy of Medical Sciences (approval number: DWSP201804001).

\section{AUTHOR CONTRIBUTIONS}

HL, ZY, and SM conceived the study and drafted the paper, HL, MZ, SC, and DX performed the experiments. HS, CF, and $\mathrm{ZY}$ helped to interpret results and contributed to the writing. All authors contributed to the article and approved the submitted version.

\section{FUNDING}

This work was supported by the Research Projects of Yunnan Province, China (Grant Number: 20200AA100009 and 2017FA006), CAMS Innovation Fund for Medical Sciences (CIFMS) (Grant Number: 2016-I2M-1-019 and 2016-I2M-3026), and Yunnan Leading Medical Scientist Training Program (Grant Number: L-2018003).

\section{SUPPLEMENTARY MATERIAL}

The Supplementary Material for this article can be found online at: https://www.frontiersin.org/articles/10.3389/fcimb.2021. 700191/full\#supplementary-material

Brown, B. A., Oberste, M. S., Alexander, J. P. Jr., Kennett, M. L., and Pallansch, M. A (1999). Molecular Epidemiology and Evolution of Enterovirus 71 Strains Isolated From 1970 to 1998. J. Virol. 73, 9969-9975. doi: 10.1128/jvi.73.12.9969-9975.1999

Chavarria-Miró, G., de Castellarnau, M., Fuentes, C., D'Andrea, L., PérezRodríguez, F. J., Beguiristain, N., et al. (2021). Advances for the Hepatitis A Virus Antigen Production Using a Virus Strain With Codon Frequency Optimization Adjustments in Specific Locations. Front. Microbiol. 12, 642267. doi: 10.3389/fmicb.2021.642267

Drago, F., Ciccarese, G., Broccolo, F., Rebora, A., and Parodi, A. (2017). Atypical Hand, Foot, and Mouth Disease in Adults. J. Am. Acad. Dermatol. 77, e51-e56. doi: 10.1016/j.jaad.2017.03.046

Du, Z., Zhao, Y., Luo, Y., Du, L., Gan, Q., Zhang, H., et al. (2019). Ongoing Change of Severe Hand, Foot, and Mouth Disease Pathogens in Yunnan, China 2012 to 2016. J. Med. Virol. 91, 881-885. doi: 10.1002/jmv.25393

Fujimoto, T. (2018). [Hand-Foot-And-Mouth Disease, Aseptic Meningitis, and Encephalitis Caused by Enterovirus]. Brain Nerve 70, 121-131. doi: 10.11477/ mf.1416200964

Fu, X., Wan, Z., Li, Y., Hu, Y., Jin, X., and Zhang, C. (2020). National Epidemiology and Evolutionary History of Four Hand, Foot and Mouth Disease-Related Enteroviruses in China From 2008 to 2016. Virol. Sin. 35, 21-33. doi: 10.1007/s12250-019-00169-2 
Hayman, R., Shepherd, M., Tarring, C., and Best, E. (2014). Outbreak of Variant Hand-Foot-and-Mouth Disease Caused by Coxsackievirus A6 in Auckland, New Zealand. J. Paediatr. Child Health 50, 751-755. doi: 10.1111/jpc.12708

He, S., Chen, M., Wu, W., Yan, Q., Zhuo, Z., Su, X., et al. (2018). An Emerging and Expanding Clade Accounts for the Persistent Outbreak of Coxsackievirus A6Associated Hand, Foot, and Mouth Disease in China Since 2013. Virology 518, 328-334. doi: 10.1016/j.virol.2018.03.012

Hoa-Tran, T. N., Dao, A. T. H., Nguyen, A. T., Kataoka, C., Takemura, T., Pham, C. H., et al. (2020). Coxsackieviruses A6 and A16 Associated With Hand, Foot, and Mouth Disease in Vietnam 2008-2017: Essential Information for Rational Vaccine Design. Vaccine 38, 8273-8285. doi: 10.1016/j.vaccine.2020.11.031

Li, Y., Chang, Z., Wu, P., Liao, Q., Liu, F., Zheng, Y., et al. (2018). Emerging Enteroviruses Causing Hand, Foot and Mouth Disease, China 2010-2016. Emerg. Infect. Dis. 24, 1902-1906. doi: 10.3201/eid2410.171953

Liu, H., Cong, S., Xu, D., Lin, K., Huang, X., Sun, H., et al. (2020a). Characterization of a Novel Echovirus 21 Strain Isolated From a Healthy Child in China in 2013. Arch. Virol. 165, 757-760. doi: 10.1007/s00705-019-04506-4

Liu, S. L., Pan, H., Liu, P., Amer, S., Chan, T. C., Zhan, J., et al. (2015). Comparative Epidemiology and Virology of Fatal and Nonfatal Cases of Hand, Foot and Mouth Disease in Mainland China From 2008 to 2014. Rev. Med. Virol. 25, 115-128. doi: 10.1002/rmv.1827

Liu, H., Zhang, J., Xu, D., Cong, S., Huang, X., Sun, H., et al. (2020b). Molecular Characteristics of a Coxsackievirus A6 Strain RYN-A1205 and its Genetic Stability in KMB17 Cells. Chin. J. Viral Dis. 10, 102-108. doi: 10.16505/j.20950136.2019 .0092

Lott, J. P., Liu, K., Landry, M. L., Nix, W. A., Oberste, M. S., Bolognia, J., et al. (2013). Atypical Hand-Foot-and-Mouth Disease Associated With Coxsackievirus A6 Infection. J. Am. Acad. Dermatol. 69, 736-741. doi: 10.1016/j.jaad.2013.07.024

Mirand, A., le Sage, F. V., Pereira, B., Cohen, R., Levy, C., Archimbaud, C., et al. (2016). Ambulatory Pediatric Surveillance of Hand, Foot and Mouth Disease as Signal of an Outbreak of Coxsackievirus A6 Infections, France 2014-2015. Emerg. Infect. Dis. 22, 1884-1893. doi: 10.3201/eid2211.160590

Montes, M., Artieda, J., Piñeiro, L. D., Gastesi, M., Diez-Nieves, I., and Cilla, G. (2013). Hand, Foot, and Mouth Disease Outbreak and Coxsackievirus A6, Northern Spain 2011. Emerg. Infect. Dis. 19, 676-678. doi: 10.3201/eid1904.121589

Nishimura, Y., Shimojima, M., Tano, Y., Miyamura, T., Wakita, T., and Shimizu, H. (2009). Human P-Selectin Glycoprotein Ligand-1 Is a Functional Receptor for Enterovirus 71. Nat. Med. 15, 794-797. doi: 10.1038/nm.1961

Prim, N., Rodriguez, G., Margall, N., Del Cuerpo, M., Trallero, G., and Rabella, N. (2013). Combining Cell Lines to Optimize Isolation of Human Enterovirus From Clinical Specimens: Report of 25 Years of Experience. J. Med. Virol. 85, 116-120. doi: 10.1002/jmv.23426

Ramirez-Fort, M. K., Downing, C., Doan, H. Q., Benoist, F., Oberste, M. S., Khan, F., et al. (2014). Coxsackievirus A6 Associated Hand, Foot and Mouth Disease in Adults: Clinical Presentation and Review of the Literature. J. Clin. Virol. 60, 381-386. doi: 10.1016/j.jcv.2014.04.023

Shin, J. U., Oh, S. H., and Lee, J. H. (2010). A Case of Hand-Foot-Mouth Disease in an Immunocompetent Adult. Ann. Dermatol. 22, 216-218. doi: 10.5021/ ad.2010.22.2.216

Solomon, T., Lewthwaite, P., Perera, D., Cardosa, M. J., McMinn, P., and Ooi, M. H. (2010). Virology, Epidemiology, Pathogenesis, and Control of Enterovirus 71. Lancet Infect. Dis. 10, 778-790. doi: 10.1016/s1473-3099(10)70194-8

Song, Y., Zhang, Y., Ji, T., Gu, X., Yang, Q., Zhu, S., et al. (2017). Persistent Circulation of Coxsackievirus A6 of Genotype D3 in Mainland of China Between 2008 and 2015. Sci. Rep. 7, 5491. doi: 10.1038/s41598-017-05618-0

Weng, Y., Chen, W., Huang, M., He, W., Zheng, K., and Yan, Y. (2017). Epidemiology and Etiology of Hand, Foot, and Mouth Disease in Fujian Province 2008-2014. Arch. Virol. 162, 535-542. doi: 10.1007/s00705-016-3127-x

Woods, G. L., and Young, A. (1988). Use of A-549 Cells in a Clinical Virology Laboratory. J. Clin. Microbiol. 26, 1026-1028. doi: 10.1128/jcm.26.5.10261028.1988

Xing, W., Liao, Q., Viboud, C., Zhang, J., Sun, J., Wu, J. T., et al. (2014). Hand, Foot, and Mouth Disease in China 2008-12: An Epidemiological Study. Lancet Infect. Dis. 14, 308-318. doi: 10.1016/s1473-3099(13)70342-6
Xu, W., and Zhang, Y. (2016). Isolation and Characterization of Vaccine-Derived Polioviruses, Relevance for the Global Polio Eradication Initiative. Methods Mol. Biol. 1387, 213-226. doi: 10.1007/978-1-4939-3292-4_10

Xu, L., Zheng, Q., Li, S., He, M., Wu, Y., Li, Y., et al. (2017). Atomic Structures of Coxsackievirus A6 and its Complex With a Neutralizing Antibody. Nat. Commun. 8, 505. doi: 10.1038/s41467-017-00477-9

Yamayoshi, S., Yamashita, Y., Li, J., Hanagata, N., Minowa, T., Takemura, T., et al. (2009). Scavenger Receptor B2 Is a Cellular Receptor for Enterovirus 71. Nat. Med. 15, 798-801. doi: 10.1038/nm.1992

Yang, L., Mao, Q., Li, S., Gao, F., Zhao, H., Liu, Y., et al. (2016). A Neonatal Mouse Model for the Evaluation of Antibodies and Vaccines Against Coxsackievirus A6. Antiviral Res. 134, 50-57. doi: 10.1016/j.antiviral.2016.08.025

Yang, F., Yuan, J., Wang, X., Li, J., Du, J., Su, H., et al. (2014). Severe Hand, Foot, and Mouth Disease and Coxsackievirus A6-Shenzhen, China. Clin. Infect. Dis. 59, 1504-1505. doi: 10.1093/cid/ciu624

Yi, E. J., Shin, Y. J., Kim, J. H., Kim, T. G., and Chang, S. Y. (2017). Enterovirus 71 Infection and Vaccines. Clin. Exp. Vaccine Res. 6, 4-14. doi: 10.7774/ cevr.2017.6.1.4

Zeng, H., Lu, J., Yang, F., Liu, L., Zheng, H., Ke, C., et al. (2018). The Increasing Epidemic of Hand, Foot, and Mouth Disease Caused by Coxsackievirus-A6, Guangdong, China 2017. J. Infect. 76, 220-223. doi: 10.1016/j.jinf.2017.11.005

Zhang, Z., Dong, Z., Wei, Q., Carr, M. J., Li, J., Ding, S., et al. (2017). A Neonatal Murine Model of Coxsackievirus A6 Infection for Evaluation of Antiviral and Vaccine Efficacy. J. Virol. 91, e02450-e02416. doi: 10.1128/jvi.02450-16

Zhang, B., Wu, X., Huang, K., Li, L., Zheng, L., Wan, C., et al. (2014). The Variations of VP1 Protein Might Be Associated With Nervous System Symptoms Caused by Enterovirus 71 Infection. BMC Infect. Dis. 14, 243. doi: 10.1186/1471-2334-14-243

Zhang, H., Zhang, L., Xie, J., Wen, W., Wei, L., and Nie, B. (2019). Probiotics Supplement in Children With Severe Hand, Foot, and Mouth Disease: A Systematic Review and Meta-Analysis Protocol. Med. (Baltimore) 98, e17939. doi: $10.1097 / \mathrm{md} .0000000000017939$

Zhang, M., Zhao, Y., Zhang, H., Lin, K., Liu, H., Zhang, J., et al. (2019). Molecular Characterization of Coxsackievirus A16 Strains Isolated From Children With Severe Hand, Foot, and Mouth Disease in Yunnan, Southwest China, During 2009-2015. J. Med. Virol. 91, 155-160. doi: 10.1002/jmv.25297

Zhou, Y., Shen, C., Zhang, C., Zhang, W., Wang, L., Lan, K., et al. (2016). YeastProduced Recombinant Virus-Like Particles of Coxsackievirus A6 Elicited Protective Antibodies in Mice. Antiviral Res. 132, 165-169. doi: 10.1016/ j.antiviral.2016.06.004

Zhu, L., Sun, Y., Fan, J., Zhu, B., Cao, L., Gao, Q., et al. (2018). Structures of Coxsackievirus A10 Unveil the Molecular Mechanisms of Receptor Binding and Viral Uncoating. Nat. Commun. 9, 4985. doi: 10.1038/s41467-018-07531-0

Zhu, F., Xu, W., Xia, J., Liang, Z., Liu, Y., Zhang, X., et al. (2014). Efficacy, Safety, and Immunogenicity of an Enterovirus 71 Vaccine in China. $N$ Engl. J. Med. 370, 818-828. doi: 10.1056/NEJMoa1304923

Conflict of Interest: The authors declare that the research was conducted in the absence of any commercial or financial relationships that could be construed as a potential conflict of interest.

Publisher's Note: All claims expressed in this article are solely those of the authors and do not necessarily represent those of their affiliated organizations, or those of the publisher, the editors and the reviewers. Any product that may be evaluated in this article, or claim that may be made by its manufacturer, is not guaranteed or endorsed by the publisher.

Copyright $\odot 2021$ Liu, Zhang, Feng, Cong, Xu, Sun, Yang and Ma. This is an openaccess article distributed under the terms of the Creative Commons Attribution License (CC BY). The use, distribution or reproduction in other forums is permitted, provided the original author(s) and the copyright owner(s) are credited and that the original publication in this journal is cited, in accordance with accepted academic practice. No use, distribution or reproduction is permitted which does not comply with these terms. 